\title{
Tissue inhibitor of metalloproteinases-2 gene polymorphisms in chronic obstructive pulmonary disease
}

\author{
K. Hirano, T. Sakamoto, Y. Uchida, Y. Morishima, K. Masuyama, Y. Ishii, A. Nomura, \\ M. Ohtsuka, K. Sekizawa
}

Tissue inhibitor of metalloproteinases-2 gene polymorphisms in chronic obstructive pulmonary disease. K. Hirano, T. Sakamoto, Y. Uchida, Y. Morishima, K. Masuyama, Y. Ishii, A. Nomura, M. Ohtsuka, K. Sekizawa. (C) ERS Journals Ltd 2001.

ABSTRACT: Proteinase/antiproteinase imbalance is the most widely accepted theory for development of chronic obstructive pulmonary disease (COPD). Mutations of tissue inhibitor of metalloproteinases-2 (TIMP-2) that downregulate its activity may increase the activities of matrix metalloproteinases and result in the degradation of the lung matrix.

Polymorphisms of the TIMP-2 gene were investigated in 88 COPD patients and 40 control subjects. The variations were examined by single-strand conformational polymorphism analysis followed by sequencing.

Two polymorphisms were identified, +853 G/A and -418 G/C nucleotide substitutions. There was a significant deviation in the genotypic frequencies at +853 and the allele frequencies for $G$ were significantly higher in the COPD patient group than in the control group. For locus $\mathbf{- 4 1 8}$, the allele frequencies for $\mathrm{C}$ in the COPD patient group also tended to be higher than those in the control group. The +853 G/A nucleotide substitution was a silent variant. The $\mathbf{- 4 1 8}$ G/C substitution was located in the consensus sequence for the Sp1 binding site.

These polymorphisms may be associated with the development of chronic obstructive pulmonary disease, decreasing the transcription and stability of the messenger ribonucleic acid, and available as genetic markers of susceptibility to the disease. Eur Respir J 2001; 18: 748-752.
Dept of Pulmonary Medicine, Institute of Clinical Medicine, University of Tsukuba, Ibaraki, Japan.

Correspondence: Y. Uchida, Dept of Pulmonary Medicine, Institute of Clinical Medicine, University of Tsukuba, 1-1-1 Tennnoudai Tsukuba, Ibaraki 305-8575, Japan.

Fax: 81298533144

Keywords: Chronic obstructive pulmonary disease

polymorphism

tissue inhibitors of metalloproteinases

Received: December 62000

Accepted after revision April 32001
Chronic obstructive pulmonary disease (COPD) is characterized by a slowly progressive airflow limitation caused by emphysema and/or airway disease. The most important risk factor for COPD is cigarette smoke and nearly $90 \%$ of COPD patients are smokers [1]. Cigarette smoke contains high concentrations of free radicals, which recruit inflammatory cells such as neutrophils and macrophages to the lung. These inflammatory cells also generate reactive oxygen species and release a variety of proteolytic enzymes. The oxidants inactivate antiproteases such as $\alpha-1$ proteinase inhibitor, antileukoprotease and tissue inhibitors of metalloproteinases (TIMPs) [2-4] as well as directly damage the lung matrix [5]. These events generate the proteinase/antiproteinase imbalance that is the most widely accepted theory for the pathogenesis of COPD. However, only $\sim 15 \%$ of cigarette smokers develop COPD. Therefore, it is suggested that genetic susceptibility to smoking injury may contribute to the development of COPD. This hypothesis is also supported by evidence that there are differences in the prevalence of COPD in smokers between different ethnic groups [6]. The genetic factors determining the susceptibility might depend on increased activities of proteolytic enzymes on the one hand, or inactivation of protective enzymes against oxidative or proteolytic injuries on the other.
Recently, several studies have demonstrated that expressions or activities of matrix metalloproteinases (MMPs), especially MMP-1, 2, 8, 9 and membranetype MMP-1 (MT1-MMP), are elevated in the lung of COPD patients [7-10]. These findings suggest that upregulated activities of MMPs play a role in the pathogenesis of COPD. One important mechanism for the regulation of the activities of MMPs is the binding to TIMPs. Four members of the TIMP family (TIMP1 to TIMP-4) interact with active forms of MMPs to inhibit their activities. However, it is reported that TIMP-4 messenger ribonucleic acid (mRNA) cannot be detected in the lung [11]. And it is mainly TIMP-1 and 2 that have been shown to participate in pulmonary diseases characterized by alterations of alveolar structure or abnormal remodelling responses such as emphysema, interstitial fibrosis, acute respiratory distress syndrome and lung cancer. Furthermore, it has been suggested that the MT1-MMP/MMP-2/ TIMP-2 system plays a significant role in the formation of pulmonary emphysema [8]. Therefore, it is possible that mutations of the TIMP-2 gene that downregulate its own activity result in the progression of the breakdown of lung matrix.

In this study, genetic variations in the promoter and coding regions of the TIMP-2 gene were investigated. Two polymorphisms were identified; one in the 
promoter region and the other in exon 3, and an association analysis was carried out for these polymorphisms in COPD patients and healthy controls. Possible mechanisms by which these variants inhibit the activity of TIMP-2 are discussed.

\section{Subjects and methods}

\section{Subjects}

Eighty-eight patients with COPD (85 males and three females) and 40 healthy, age-matched volunteers (all males) were studied (table 1). The diagnosis of COPD was based on a (forced expiratory volume in one second (FEV1) of $<70 \%$ predicted for age and height as well as a FEV1/FVC ratio of $<70 \%$; a chest radiograph that showed hyperinflation, flattened diaphragms, and a marked loss of vascularity; and an absence of a history of bronchial asthma, bronchiectasis, or other significant respiratory diseases. The subjects were asked to avoid using a bronchodilator for at least $4 \mathrm{~h}$ before the spirometry, unless respiratory symptoms required the treatment. Written informed consent was obtained from all subjects according to the research protocol approved by the Ethics Committee of the University of Tsukuba.

\section{Deoxyribonucleic acid samples and polymerase chain reaction conditions}

Genomic deoxyribonucleic acid (DNA) samples were prepared from whole blood using a QIAGEN DNA blood kit (QIAGEN, Hilden, Germany). The nucleotide sequence and the genomic structure of the human TIMP-2 gene have been reported previously [12], (Genbank accession number U44381-U44385). The positions of nucleotides in this study are given relative to the major transcription initiation site. Eleven sets of oligonucleotide primers were prepared to produce polymerase chain reaction (PCR) DNA fragments covering the promoter region and the whole coding region (table 2). The $5^{\prime}$ terminus of each primer was labelled with fluorescein isothiocyanate for fluorescence-based single-strand conformational polymorphism (SSCP) analysis. PCR amplification was performed with $100 \mathrm{ng}$ of genomic DNA in a total volume of $50 \mu \mathrm{L}$ containing $200 \mu \mathrm{M}$ of each deoxyribonucleoside triphosphate (dNTP), $100 \mathrm{nM}$ of each primer, and $1.25 \mathrm{U}$ of Hotstar Taq DNA polymerase (QIAGEN). The annealing temperatures for PCR are given in table 2 .

Single-strand conformational polymorphism analysis and sequencing of polymerase chain reaction products

A DNA sequencer, ALF II (Amersham Pharmacia Biotech, Uppsala, Sweden), was used to perform fluorescence-based SSCP analysis. Three microlitres of the PCR product was added to $20 \mu \mathrm{L}$ of a loading solution consisting of $99.5 \%$ deionized formamide and $0.5 \%$ blue dextran. After denaturation at $96^{\circ} \mathrm{C}$ for $5 \mathrm{~min}, 3 \mu \mathrm{L}$ of the mixture was applied to a $7 \%(49: 1$, acrylamide:bisacrylamide ratio) or a $10 \%$ (99:1, acrylamide:bisacrylamide ratio) polyacrylamide gel containing $0.5 \times$ Tris-Borate-ethylenediamine tetraacetic acid (EDTA) buffer. Electrophoresis was performed at $20 \mathrm{~W}$ for $4 \mathrm{~h}$ at $18^{\circ} \mathrm{C}$. PCR products showing variant band patterns by SSCP were separated in $1.2 \%$ agarose gel and then extracted from the gel. DNA sequences of the PCR products were directly determined by the dideoxynucleotide chaintermination method with a dideoxyterminator kit (Perkin Elmer Applied Biosystems, Foster City, CA, USA) and a DNA sequencer model 373S (Perkin Elmer Applied Biosystems).

\section{Statistical analysis}

The values in the profile shown in table 1 are the mean \pm sEM. Statistical analysis of age, Brinkman's index (the number of cigarettes $\cdot \mathrm{day}^{-1} \times$ the number of yrs) and results of the pulmonary function test was performed by unpaired t-test. Significance was accepted at $\mathrm{p}<0.05$. Significant differences in both genotypic and allele frequencies between patients and controls were analysed by using Fisher's exact test $(2 \times 3$ table and $2 \times 2$ table $)$. Odds ratios and $95 \%$ confidence intervals (CIs) were calculated to quantitatively assess the degree of association between particular alleles and COPD.

Table 1. - Clinical features of chronic obstructive pulmonary disease (COPD) patients and controls

\begin{tabular}{lccc}
\hline & Patients with COPD & Control subjects & p-value \\
\hline Subjects n & 88 & 40 & \\
Sex M:F & $88: 3$ & $40: 0$ & NS \\
Age yrs & $66.9 \pm 1.6$ & $72.9 \pm 1.0$ & $\mathrm{NS}$ \\
Brinkman's index & $1201.6 \pm 70.1$ & $1071.1 \pm 66.5$ & $<0.0001$ \\
Pulmonary function & & & $<0.0001$ \\
FVC\% pred & $77.4 \pm 2.2$ & $95.9 \pm 3.8$ & $<0.0001$ \\
FEV1 L & $1.1 \pm 0.1$ & $2.4 \pm 1.4$ & $85.1 \pm 1.6$ \\
FEV1/FVC\% & $47.1 \pm 1.3$ & & \\
\hline
\end{tabular}

Data are presented as the mean \pm SEM. Brinkman's index: the number of cigarettes $\cdot$ day $^{-1} \times$ the number of yrs; FVC: forced vital capacity; FEV1: forced expiratory volume in one second; NS: nonsignificant. 
Table 2. - Oligonucleotide primers for amplification of the promoter region and the whole coding region of the human tissue inhibitor of metalloproteinases-2 gene

\begin{tabular}{|c|c|c|c|c|}
\hline Name & Sequence & Position & Size bp & Annealing temperature ${ }^{\circ} \mathrm{C}$ \\
\hline TP-2PF1 & 5'-AGTAGCTGGGACTACAGGCG-3' & $-1210--1191$ & 303 & 68.0 \\
\hline TP-2PR 1 & 5'-GGCTCCATGGACACTGCTGC-3' & $-927--908$ & & \\
\hline TP-2PF2 & 5'-GAGGGCTGGAGTGCTGACCT-3' & $-951--932$ & 319 & 64.0 \\
\hline TP-2PR2 & 5'-TGACCAGCCAACAAGAGACG-3' & $652--633$ & & \\
\hline TP-2PF3 & 5'-CGTCTCTTGTTGGCTGGTCA-3' & $-652--633$ & 304 & 61.0 \\
\hline TP-2PR 3 & 5'-CCTTCAGCTCGACTCTGGAG-3' & $-368--349$ & & \\
\hline TP-2PF4 & 5'-GGACGGAGCAGCGTAGCCCT-3' & $-386--367$ & 308 & 68.0 \\
\hline TP-2PR4 & 5'-CTCTGGGCGCTCCCAGCAGC-3' & $-98--79$ & & \\
\hline TP-2PF5 & 5'-CACAACAAAAGCGCGGGCTG-3' & $-149--130$ & 292 & 56.0 \\
\hline TP-2PR5 & 5'-TTCТCТССТСТTTGTCTCGG-3' & $+123-+142$ & & \\
\hline $\mathrm{TP}-2 \mathrm{~F} 1$ & 5'-CCGAGACAAAGAGGAGAGAA-3' & $+123-+142$ & 326 & 56.0 \\
\hline TP-2R1 & 5'-CTGGGGTCGCCCGACTCCTT-3' & $+429-+448$ & & \\
\hline $\mathrm{TP}-2 \mathrm{~F} 2$ & 5'-CAGGAGCGTGTTTCAGGAAC-3' & $+452-+471$ & 246 & 59.0 \\
\hline TP-2R2 & 5'-CAGATCGTGTTCCAGGGACC-3' & $+678-+697$ & & \\
\hline TP-2F3 & 5'-GCCCAGGGTGTTCTGGATGG-3' & $+699-+718$ & 221 & 66.0 \\
\hline TP-2R3 & 5'-CTCCGGCTGATGGCCCCACT-3' & $+900-+919$ & & \\
\hline TP-2F4 & 5'-CTGAGCTCGGTGATCCCCGT-3' & $+1087-+1106$ & 276 & 55.0 \\
\hline TP-2R4 & 5'-CTGGTCTTAGATCAGGAGGC-3' & $+1343-+1362$ & & \\
\hline TP-2F5A & 5'-CCTTCCTGGGTCCCTGACTC-3' & $+1960-+1979$ & 303 & 62.0 \\
\hline TP-2R5A & 5'-CAGTCGAAACCCTTGGAGGC-3' & $+2243-+2262$ & & \\
\hline TP-2F5B & 5'-ATCGAGGACCCATAAGCAGG-3' & $+2190-+2209$ & 353 & 60.0 \\
\hline TP-2R5B & 5'-GAGATCCCTAAGTGCTGCCT-3' & $+2523-+2542$ & & \\
\hline
\end{tabular}

bp: base pairs.

\section{Results}

Table 1 demonstrates the characteristics of the subjects. All patients and controls were smokers and ethnically homogenous Japanese. All the 88 patients had moderate to severe COPD and the FEV1/FVC ratio was $47.1 \pm 1.3 \%$. Age and Brinkman's index were not significantly different between the patient group and the control group.

Two variants for the TIMP-2 gene were identified by PCR-SSCP analysis followed by direct DNA sequencing (fig.1). One was a G/A nucleotide substitution at position +853 in exon 3 that was a silent variant. The other was characterized by a single base G/C nucleotide substitution at position -418 , which was located in the consensus sequence for Spl (GAGGCTGGG) in the promoter region. The homozygous wild-type, the homozygous variant-type, and the heterozygous genotype were identified for these two variants. Since no restriction enzyme was available for these polymorphisms, SSCP analysis was used to screen for the presence or absence of the nucleotide substitution.

Table 3 shows the genotypic and allele frequencies at position +853 and -418 both in COPD patients and controls. As for the polymorphism at position +853 , distributions of 40 genotypes in the controls were 24 (60.0\%) for $\mathrm{G} / \mathrm{G}, 14(35.0 \%)$ for $\mathrm{G} / \mathrm{A}$ and $2(5.0 \%)$ for A/A. Conversely, distributions of 88 genotypes in the COPD patients were $79(89.7 \%)$ for $\mathrm{G} / \mathrm{G}, 9(10.2 \%)$ for a)

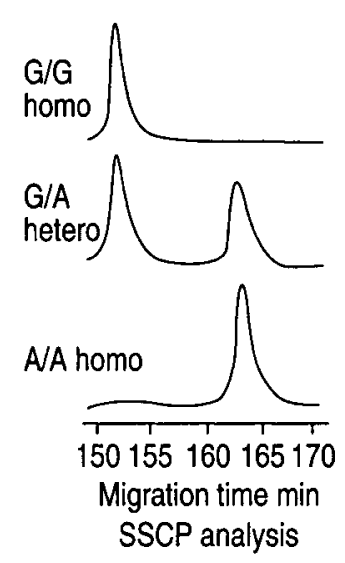

G G TCTCG CTGGA
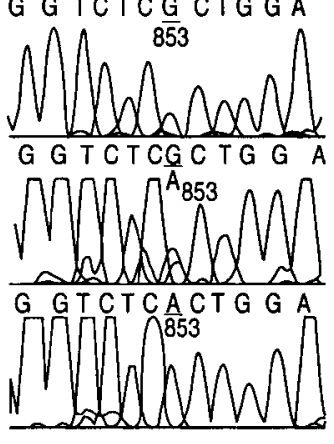

Sequencing b)

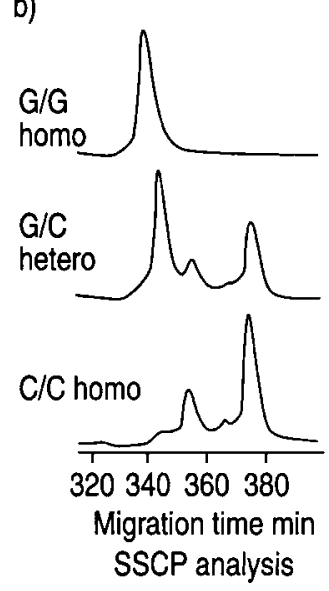

G A G G CT G G G

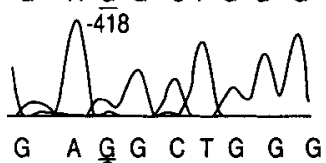

G $A G G C T G G G$

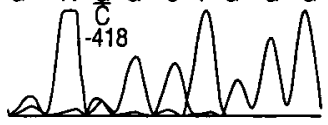

$G A \subseteq G C T G G G$

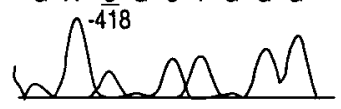

Sequencing

Fig. 1. - Single-strand conformational polymorphism (SSCP) analysis and direct sequencing of polymerase chain reaction (PCR) products generated with the primer sets a) TP-2F3/TP-2R3; and b) TP-2PF3/TP-2PR3. a) Single base A/G nucleotide substitution at position +853 ; b) single base G/C nucleotide substitution. Both of the homozygous and the heterozygous genotypes were identified at each position. 
Table 3. - Genotypic and allele frequencies at +853 and -418 loci of tissue inhibitor of metalloproteinases- 2 gene in chronic obstructive pulmonary disease (COPD) patients and control subjects

\begin{tabular}{|c|c|c|c|c|c|c|c|c|c|c|}
\hline \multirow[t]{3}{*}{ Subjects } & \multicolumn{5}{|c|}{+853 locus } & \multicolumn{5}{|c|}{-418 locus } \\
\hline & \multicolumn{3}{|c|}{ Genotype } & \multicolumn{2}{|c|}{ Allele } & \multicolumn{3}{|c|}{ Genotype } & \multicolumn{2}{|c|}{ Allele } \\
\hline & $\mathrm{G} / \mathrm{G}$ & G/A & $\mathrm{A} / \mathrm{A}$ & G & A & $\mathrm{G} / \mathrm{G}$ & $\mathrm{G} / \mathrm{C}$ & $\mathrm{C} / \mathrm{C}$ & G & $\mathrm{C}$ \\
\hline COPD & 79 (89.7) & $9(10.2)$ & $0(0.0)$ & 167 (94.9) & $9(5.1)$ & $54(61.3)$ & $32(36.4)$ & $2(2.3)$ & $140(79.5)$ & $36(20.5)$ \\
\hline Control & $24(60.0)$ & $14(35.0)$ & $2(5.0)$ & $62(77.5)$ & $18(22.5)$ & $33(82.5)$ & $6(15.0)$ & $1(2.5)$ & $72(90.0)$ & $8(10.0)$ \\
\hline $\begin{array}{l}\text { p-Value } \\
\text { Odds ratio } \\
\quad(95 \% \mathrm{CI})\end{array}$ & & 0.0001 & & \multicolumn{2}{|c|}{$\begin{array}{c}<0.0001 \\
5.4(2.3-12.6)\end{array}$} & & 0.056 & & \multicolumn{2}{|c|}{$2.3(1.0-5.2)$} \\
\hline
\end{tabular}

Data presented as n (\%) unless otherwise stated. CI: confidence interval.

G/A and $0(0 \%)$ for $\mathrm{A} / \mathrm{A}$. There was a significant deviation in these genotypic frequencies between the COPD patient group and the control group $(\mathrm{p}=$ $0.0001)$. In addition, the allele frequency for $G$ at position +853 was significantly higher in the COPD patients than in the control subjects $(94.9 \%$ in COPD patients versus $77.5 \%$ in controls; $\mathrm{p}<0.0001)$. For the patients with COPD, the odds ratio for $\mathrm{G}$ at +853 versus the control subjects was 5.4 (95\% CI 2.3-12.6). In regard to the single base $\mathrm{G} / \mathrm{C}$ substitution at position -418, the genotypic and allele frequencies were not significantly different between the two groups by both-side analysis of the Fisher's exact test. However, since the p-value for the allele frequencies was 0.049 and the odds ratio for the difference in the distribution was 2.3 (95\% CI 1.0-5.2), it seems that the nucleotide substitution of $\mathrm{C}$ for $\mathrm{G}$ at position -418 tends to occur in the COPD patients. There was no positive correlation between the variants at position +853 and those at the Spl binding site at position -418 .

\section{Discussion}

Two polymorphisms in the TIMP-2 gene were identified. One was the $+853 \mathrm{G} / \mathrm{A}$ nucleotide substitution in exon 3 , and the other was the G/C nucleotide substitution at position -418 in the promoter region. The former polymorphism, the nucleotide substitution of $\mathrm{G}$ for $\mathrm{A}$ at position +853 , has been identified in patients with aneurysms [13]; the allele frequencies for $G$ were significantly higher in male patients with abdominal aortic aneurysm than in controls. In the present study, the majority of subjects were male. There might be differences between the sexes in susceptibility to disease in subjects with this polymorphism. It has also been demonstrated that patients with severe COPD have a significantly higher prevalence of aneurysms compared to mild or moderate COPD patients [14]. Therefore, this polymorphism might be conserved for both COPD and aneurysms. Although the +853 G/A nucleotide substitution does not change the amino acid, it is possible that this polymorphism is related to the downregulation of TIMP-2 activity that leads to degradation of the structural matrix and results in development of both COPD and aortic aneurysms. One possibility is that the polymorphism is in linkage disequilibrium with an original causal variant, which the authors could not identify in this study. Although the sensitivity of SSCP analysis is very high $(80-90 \%)$ [15], it is possible that some variants cannot be detected. It is also likely that the original causal variant was simply not within the region screened. Another possibility is that this nucleotide substitution exerts an influence on the secondary structure of the mRNA, which inhibits the rate of translation by interfering with ribosome binding and/or decreases the mRNA stability.

Another polymorphism, the single base G/C substitution at position -418, is located in the consensus sequence for the Sp1 binding site [16] in the promoter region. Spl protein binds to the consensus sequence in a variety of cells and stimulates transcriptional activity. A study of the promoter activity of the TIMP-2 gene has demonstrated that a 519-bp segment upstream from the major transcription initiation site contains active promoter elements [17]. Therefore, it is possible that the -418 nucleotide substitution in the consensus sequence for $\mathrm{Sp} 1$ results in downregulation of the transcriptional activity of the TIMP-2 gene.

There is evidence that MMPs, especially MMP-1, 2, 8, 9 and MT1-MMP, play a role in the development of COPD. The mRNA expressions or the enzymatic activities of the MMPs are elevated in alveolar macrophages, the lung tissue or bronchoalveolar lavage fluid from COPD patients [7-9]. Immunoreactivity for the MMPs is observed in the lung tissue of COPD patients [8, 10]. Furthermore, it has been reported that macrophage elastase (MME; MMP-12) gene-knockout mice $\left(\mathrm{MME}^{-/-}\right)$do not develop emphysema in response to exposure to cigarette smoke, while wild-type $\left(\mathrm{MME}^{+/+}\right)$mice do [18]. Their study directly demonstrated the contribution of MMP-12 to the pathogenesis of cigarette smoke-induced emphysema. All of these MMPs are counteracted by TIMPs. Until now, no comprehensive quantitative comparison has been made of the inhibitory selectivity of different TIMPs with different MMPs. However, it is suggested that the MT1-MMP/MMP-2/TIMP-2 system is important in extracellular matrix degradation of emphysematous lung [8]. In addition, TIMP-2 is a more effective inhibitor of MMP-2 and MMP-9 than TIMP-1 [19]. It seems likely that downregulation of TIMP-2 leads to increased activities of MMPs and results in the development of COPD. 
COPD is a heterogeneous disease including emphysema and chronic bronchitis. MMPs break down connective tissue in the lung parenchyma resulting in emphysema. In contrast, chronic bronchitis is an inflammatory disease characterized by hypersecretion from hypertrophic bronchial glands. It is suggested that the secretion and activation of MMP-2 is correlated with gland morphogenesis [20]. In addition, MMPs recruit neutrophils to the lung [21], resulting in mucous hypersecretion and inflammation of the bronchial trees. Therefore, it is possible that the inhibition of TIMP-2 activity is related to chronic bronchitis as well as emphysema.

In conclusion, two polymorphisms for the tissue inhibitor of metalloproteinases- 2 gene were associated with chronic obstructive pulmonary disease susceptibility caused by cigarette smoke in a Japanese population. Although chronic obstructive pulmonary disease is thought to be caused by a complex interaction of environmental and genetic factors, the polymorphisms identified in this study might be useful for predicting the susceptibility to the disease. Quitting smoking may be critical for the prevention of chronic obstructive pulmonary disease in people with either of these polymorphisms. It is also expected that these polymorphisms will contribute to the elucidation of mechanisms for the development of chronic obstructive pulmonary disease. It would be interesting to investigate the prevalence of these polymorphisms in chronic obstructive pulmonary disease patients among different ethnic groups.

\section{References}

1. Snider GL. Chronic obstructive pulmonary disease: risk factors, pathophysiology and pathogenesis. Annu Rev Med 1989; 40: 411-429.

2. Hubbard RC, Ogushi F, Fells GA, et al. Oxidants spontaneously released by alveolar macrophages of cigarette smokers can inactivate the active site of alpha-1-antitrypsin, rendering it ineffective as an inhibitor of neutrophil elastase. J Clin Invest 1987; 80: 1289-1295.

3. Kramps JA, Rudolphus A, Stolk J, Willems LNA, Dijkman JH. Role of antileukoprotease in the human lung. Ann NY Acad Sci USA 1991; 642: 97-108.

4. Mattana J, Margiloff L, Sharma P, Singhal PC. Oxidation of the mesangial matrix metalloproteinase-2 impairs gelatinolytic activity. Inflammation 1998; 22: 269-276.

5. Cantin A, Crystal RG. Oxidants, antioxidants and the pathogenesis of emphysema. Eur J Respir Dis 1985; 66: Suppl. 139, 7-17.

6. Marcus EB, Buist AS, Curb JD, et al. Correlates of FEV1 and prevalence of pulmonary conditions in Japanese-American men. Am Rev Respir Dis 1988; 138: $1398-1404$.
7. Finlay GA, O'Driscoll LR, Russell KJ, et al. Matrix metalloproteinase expression and production by alveolar macrophages in emphysema. Am J Respir Crit Care Med 1997; 156: 240-247.

8. Ohnishi K, Takagi M, Kurokawa Y, Satomi S, Konttinen YJ. Matrix metalloproteinase-mediated extracellular matrix protein degradation in human pulmonary emphysema. Lab Invest 1998; 78: 10771087.

9. Betsuyaku T, Nishimura $\mathrm{M}$, Takeyabu $\mathrm{K}$, et al. Neutrophil granule proteins in bronchoalveolar lavage fluid from subjects with subclinical emphysema. Am J Respir Crit Care Med 1999; 159: 1985-1991.

10. Segura-Valdez L, Pardo A, Gaxiola M, Uhal BD, Bacerril C, Selman M. Upregulation of gelatinases A and $\mathrm{B}$, collagenases 1 and 2 , and increased parenchymal cell death in COPD. Chest 2000; 117: 684-694.

11. Greene J, Wang M, Liu YE, Raymond LA, Rosen C, Shi YE. Molecular cloning and characterization of human tissue inhibitor of metalloproteinase 4. J Biol Chem 1996; 271: 30375-30380.

12. Hammani K, Blakis A, Morsette D, et al. Structure and characterization of the human tissue inhibitor of metalloproteinases-2 gene. J Biol Chem 1996; 271: 25498-25505.

13. Wang X, Tromp G, Cole CW, et al. Analysis of coding sequences for tissue inhibitor of metalloproteinases 1 (TIMP1) and 2 (TIMP2) in patients with aneurysms. Matrix Biol 1999; 18: 121-124.

14. van Laahoven CJ, Borstlap AC, van Berge Henegouwen DP, Palmen FM, Verpalen MC, Schoemaker MC. Chronic obstructive pulmonary disease and abdominal aortic aneurysms. Eur J Vasc Surg 1993; 7: 386-390.

15. Jordanova A, Kalaydjieva L, Savov A, et al. SSCP analysis: a blind sensitivity trial. Hum Mutat 1997; 10 : 65-70.

16. Faisst S, Meyer S. Compilation of vertebrate-encoded transcription factors. Nucleic Acids Res 1992; 20: 3-26.

17. DeClerck YA, Darville MI, Eeckhout Y, Rousseau GG. Characterization of the promoter of the gene encoding human tissue inhibitor of metalloproteinases-2 (TIMP-2). Gene 1994; 139: 185-191.

18. Hautamaki RD, Kobayashi DK, Senior RM, Shapiro SD. Requirement for macrophage elastase for cigarette smoke-induced emphysema in mice. Science 1997; 277: 2002-2004.

19. Howard EW, Bullen EC, Banda MJ. Preferential inhibition of 72- and $92-\mathrm{kDa}$ gelatinases by tissue inhibitor of metalloproteinases-2. J Biol Chem 1991; 266: 13070-13075.

20. Infeld MD. Cell-matrix interactions in gland development in the lung. Exp Lung Res 1997; 23: 161-169.

21. Gibbs DF, Shanley TP, Warner RL, Murphy HS, Varani J, Johnson KJ. Role of matrix metalloproteinases in models of macrophage-dependent acute lung injury. Evidence for alveolar macrophage as source of proteinases. Am J Respir Cell Mol Biol 1999; 20: $1145-1154$. 